\title{
PRÁTICAS CURRICULARES COM ELEMENTOS DE INCLUSIVIDADE: UMA ANÁLISE A PARTIR DA CULTURA ESCOLAR
}

\section{CURRICULAR PRACTICES WITH ELEMENTS OF INCLUSIVITY: AN ANALYSIS BASED ON SCHOOL CULTURE}

PRÁCTICAS CURRICULARES CON ELEMENTOS DE INCLUSIVIDAD: UN ANÁLISIS A PARTIR DE LA CULTURA ESCOLAR

Amélia Maria Araújo Mesquita Doutora em Educação pela UFPA.

Genylton Odilon Rêgo da Rocha Doutor em Geografia pela USP. Docente do Programa de Pós-Graduação em Educação da UFPA.

Programa de Pós-Graduação em Educação Universidade Federal do Pará (UFPA)

Belém - PA - Brasil

Endereço:

UFPA - Cidade Universitária Profo Silveira Neto Setor Profissional - Instituto de Ciência da Educação Av. Augusto Corrêa, 01 Guamá - Belém - PA CEP: $66075-110$

E-mails: amelia.mesquita05@gmail.com genylton@gmail.com

\section{RESUMO}

No contexto da educação inclusiva e da definição de parâmetros para definir uma prática que se caracterize como tal, este texto objetiva tecer algumas reflexões sobre esses engessamentos e trazer outros elementos que nos possibilitem avançar nesse campo tão tenso e contraditório. Nesse sentido, as práticas curriculares inclusivas ganham uma dimensão substantiva, passando a ser denominadas práticas curriculares com elementos de inclusividade. Tais elementos, analisados à luz da categoria cultura escolar, trazem apontamentos interessantes para pensar a inclusão como elemento constituinte da escola sendo produzida gradualmente, rompendo com a ideia de que a inclusão é um modelo exemplar exterior à escola e que por esta deve ser alcançada. As análises trazidas são resultantes da pesquisa de campo em uma escola pública estadual localizada em Belém/PA e da pesquisa bibliográfica. Os resultados apontam que a prática curricular efetivamente inclusiva é aquela que possibilita ao aluno em situação de deficiência participar, produzir e se apropriar da cultura própria da escola.

PALAVRAS-CHAVE: Inclusão. Elementos de Inclusividade. Cultura Escolar

\section{ABSTRACT}

In the context of inclusive education and the definition of parameters to define a practice that is characterized as such, this paper offers some reflections on these issues, and brings other elements that enable us to 
move forward in what is a tense and contradictory field. In this sense, inclusive curricular practices gain a substantive dimension, and are called curricular practices with elements of inclusiveness. Such elements, analyzed in the light of the category school culture, bring interesting aspects to think about inclusion as a constituent element of the school that is gradually being produced, moving away from the idea of inclusion as an external role model to be achieved by the school. The analyses presented here are the result of field research in a public school located in Belém/PA and bibliographic research. The results show that an effectively inclusive curricular practice is one that allows students with disabilities to participate, produce and appropriate the school culture.

KEYWORDS: Inclusion. Elements of Inclusivity. School Culture.

\section{RESUMEN}

En el contexto de la educación inclusiva y de la determinación de parámetros para definir una práctica que se caracterice como tal, este texto tiene por objetivo esbozar algunas reflexiones sobre esa rigidez y aportar otros elementos que nos posibiliten avanzar en ese campo tan tenso y contradictorio. En ese sentido, las prácticas curriculares inclusivas ganan una dimensión sustantiva, pasando a ser denominadas prácticas curriculares con elementos de inclusividad. Tales elementos, analizados a la luz de la categoría cultura escolar, traen aportes interesantes para pensar la inclusión como elemento constituyente de la escuela, producida gradualmente, rompiendo con la idea de que la inclusión es un modelo ejemplar exterior a la escuela y que por esta debe ser alcanzada. Los análisis expuestos son resultantes de la investigación de campo en una escuela pública estatal ubicada en Belém/PA y de investigación bibliográfica. Los resultados señalan que la práctica curricular efectivamente inclusiva es aquella que le posibilita al alumno en situación de deficiencia participar, producir y apropiarse de la cultura propia de la escuela.

PALABRAS CLAVE: Inclusión. Elementos de Inclusividad. Cultura Escolar

\section{NOTAS INTRODUTÓRIAS}

No cenário da inclusão, as práticas curriculares passam a ser adjetivadas de inclusivas e, no decorrer de aproximadamente uma década, muitas pesquisas, entre elas as de Castro (2002), Pletsch (2009), Miotto (2010), passam a ser também adjetivas de excludentes e/ou fracassadas, o que denota o aspecto paradoxal desse processo.

No levantamento de teses e dissertações junto ao Banco de Teses da CAPES, identifiquei oitenta e quatro dissertações (mestrado) e vinte e cinco teses (doutorado) sobre práticas curriculares inclusivas, sendo que apenas vinte e três dissertações e quatro teses discutiam efetivamente a prática de professores diante da inclusão de pessoas com deficiência na escola comum de ensino. Por meio do mapeamento pude perceber que $85,2 \%$ das pesquisas concluíam que o processo de inclusão da pessoa com deficiência era marcado pela fragilidade das ações do professor e por consequência do "fracasso" desse processo.

$\mathrm{Na}$ paralela desse movimento, iniciei um processo de levantamento de dados em fontes bibliográficas e documentais de forma a compreender o que e como deve se constituir uma prática curricular inclusiva. Pude perceber que existe na discussão para a inclusão a definição de um modelo exemplar, como se a prática tivesse que seguir e atingir um padrão preestabelecido, definido tanto pela política curricular inclusiva quanto pela literatura sobre esse campo de investigação. Essa hipótese foi confirmada quando encontrei o documento da Agéncia Européia para o Desenvolvimento da Educação Especial (2005), que apresenta em seu Relatório Síntese - Educação Inclusiva e Práticas 
de Sala de Aula nos $2^{\circ}$ e $3^{\circ}$ Ciclos do Ensino Básico, pelo menos sete grupos de fatores que podem ser eficazes na Educação Inclusiva: Ensino Cooperativo, Aprendizagem Cooperativa, Resolução Cooperativa de Problemas, Grupos Heterogéneos, Abordagens de Ensino Eficaz, o Ensino por Áreas Curriculares e as Estratégias Alternativas de Aprendizagem.

Esse tipo de orientação acabou por criar um parâmetro de avaliação para se definir se uma prática curricular é ou não inclusiva. É como se a ideia de inclusão se tornasse um elemento de alcance, tornando-se modelo comparativo com a escola real.

No contraponto do trabalho de levantamento bibliográfico e documental, iniciei pesquisa exploratória em uma escola pública estadual referência em inclusão escolar no município de Belém/ PA, aqui denominada Escola Pará. Contudo, apesar de ser referência, o principal critério para seleção do lócus e dos sujeitos não foi esse, porque o que me movia era a busca por uma prática curricular considerada inclusiva pela comunidade escolar - professores, coordenadores pedagógicos, gestores e pais. Foi nessa aproximação com a escola que pude "encontrar" a professora que seria sujeito de minha pesquisa (para efeitos da pesquisa ela foi denominada Professora $L$ ). Essa professora trabalhava como uma turma do $3^{\circ}$ ano do Ensino Fundamental, com um total de dezoito alunos matriculados, desses, seis eram alunos com algum tipo de deficiência ou transtorno global de desenvolvimento.

Após esse movimento exploratório, dei início ao trabalho de coleta de dados por meio da observação da prática da professora apontada como inclusiva pela comunidade escolar. Grosso modo, essa prática pode ser caracterizada como aquilo que Zabala (1998) define como aula magistral. A organização didático-pedagógica do trabalho seguia uma sequência pouco complexa, baseada basicamente no registro do assunto copiado no quadro, explicação do conteúdo, exercício de fixação. Essas atividades eram desenvolvidas por alunos sem deficiência e por alunos em situação de deficiência que apresentavam pouco comprometimento cognitivo. Para os alunos em situação de deficiência que apresentavam comprometimento cognitivo, eram desenvolvidos trabalhos paralelos fortemente vinculados às atividades de coordenação viso-motora, alfabetização e conhecimento matemático e social.

Nesse movimento, pude compreender que os elementos que constituiriam uma prática curricular inclusiva pela literatura e pela política da inclusão eram distantes dos elementos que a comunidade escolar da Escola Pará utilizam para definir uma prática inclusiva e isso me levou à necessidade de fazer alguns deslocamentos.

\section{DESLOCAMENTOS DA PESQUISA}

O desenvolvimento desta pesquisa foi permeado por um processo de intenso diálogo entre os dados de campo e os referenciais teóricos. Esse movimento me colocou diante da necessidade do deslocamento do objeto de pesquisa - e, por conseguinte, do problema de pesquisa -, da leitura semântica de determinados termos e da busca de outros referenciais para compreender e analisar os argumentos que a comunidade escolar da Escola Pará apresentaram no reconhecimento de uma prática curricular inclusiva.

Iniciei a pesquisa exploratória na intenção de encontrar e analisar como se organizava e desenvolvia uma prática curricular inclusiva. Durante essa etapa identifiquei o sujeito e, em seguida, já no processo de coleta de dados, me deparei com uma prática com fortes marcas de uma ação tradicional, com organizações de currículos paralelos e uniformes, altamente centrados nos conteúdos disciplinares - organizações essas fortemente criticadas pela literatura da inclusão. Diante do cenário e da "cena" encontrados, minha curiosidade investigativa se deslocou da prática da professora para os argumentos daquela comunidade em reconhecê-la como inclusiva. Para tanto, tive que passar por um segundo processo, desengessar-me das certezas sobre como se desenvolveria e se caracterizaria uma prática curricular inclusiva e tentar "ver" com os olhos da comunidade o que de inclusivo estava subjacente naquela prática.

"Olhar com os olhos da comunidade" me colocava diante de outra necessidade, deixar de "ler" a prática a partir de parâmetros "predefinidos" por meio da literatura da inclusão e das políticas, como que se estivesse buscando um enquadramento que definia a inclusão como um processo formado por etapas e quanto mais etapas cumpridas, mais inclusiva seria a prática, portanto, 
precisava me desprender do ideal para entender o real. Diante disse, via-me na necessidade de sair da dimensão adjetiva de inclusiva ou não inclusiva (para não dizer fracassada) para compreender a materialidade daquela prática. Foi então que optei por trabalhar com a ideia de elementos de inclusividade, na intenção de substantivar o termo inclusivo e sinalizar a existência de "matéria" naqueles argumentos.

O deslocamento do objeto e o rearranjo semântico me colocaram diante de duas perspectivas inclusivas: a ideal, produzida pela literatura da inclusão e pela política educacional inclusiva; e a real, aquela experimentada e vivida cotidianamente pelos sujeitos da comunidade escolar da Escola Pará. Essas perspectivas tinham predominantemente pontos de distanciamento, inclusive porque a literatura e as políticas falam de uma escola para o futuro (como deve ser uma escola e uma prática inclusiva) e os sujeitos da comunidade escolar falavam de uma escola do presente (da escola que "rejeitava" e que hoje "recebe" alunos em situação deficiência).

Entre a comunidade escolar da Escola Pará, encontrei pelo menos cinco aspectos, elementos de inclusividade, que sustentavam o argumento de que a prática da Professora L era inclusiva: a percepção do aluno em situação de deficiência como aluno; o aumento do tempo pessoal no tempo institucional/respeito do tempo institucional ao tempo pessoal; a frequência e a participação nos diversos espaços da escola; a apropriação de conhecimentos propriamente escolares; e o uso de artefatos escolares.

Mas percebi que esses aspectos, grosso modo, não seriam necessariamente constituidores da escola inclusiva, mas da escola como instituição social, que possui especificidades que unificam e identificam os espaços escolares naquilo que representa hoje a escola moderna do ocidente.

Foi a partir daí que pude compreender que a prática inclusiva para aquela comunidade estava na possibilidade de os alunos em situação de deficiência participarem de um próprio da escola. Diante desse movimento "encontrei" a categoria cultura escolar.

Como categoria, a compreensão da cultura escolar só foi possível pela articulação de diferentes campos disciplinares, mais prioritariamente dos campos sociológicos e históricos. A partir dessa incursão, pude depreender que a escola possui uma cultura historicamente construída e socialmente determinada, aspecto que sustenta o assente dessa instituição entre o passado e o presente, justificando a existência de tradições, ritos, artefatos, conhecimentos, como elementos configuradores da forma escolar, compreendida como uma forma de socialização (VICENT; LAHIRE; THIN, 2001).

Foi nesse elemento essencial da escola que busquei argumentos para construir e sustentar a tese de que a prática curricular inclusiva para a comunidade da Escola Pará é aquela que possibilita ao aluno em situação de deficiência participar/produzir, apropriar-se da cultura escolar, ou seja, a prática inclusiva se desenvolveria na continuidade da cultura escolar, como próprio da escola, sendo também "disponível" aos alunos em situação de deficiência.

Para melhor compreender esse jogo, é fundamental discutir um pouco mais aquilo que defini como elementos de inclusividade e posteriormente sua articulação com a cultura escolar.

\section{OS ELEMENTOS DE INCLUSIVIDADE - DA ADJETIVAÇÃO À SUBSTANTIVAÇÃO DA PRÁTICA}

Os deslocamentos que precisei fazer foram oriundos da necessidade de experimentar o desengessar da moldura que buscava enquadrar a professora num modelo (o modelo de prática curricular inclusiva definido pela literatura e pela política da inclusão). Esse desengessamento me colocava diante da fluidez do "modelo" de inclusão, assim como da compreensão de que o seu significado não é polissêmico apenas por ter definições diferentes advindas das políticas ou dos diferentes campos teóricos. A polissemia do significado de inclusão está também nas necessidades e nas expectativas dos sujeitos que a experimentam. Sujeitos reais, vivos, marcados por histórias, por contextos sociais e culturais diversificados.

Assim, mais do que construir um juízo de valor sobre a prática daquela professora (qualificando-a [a prática] como inclusiva), construído a partir de parâmetros abstratos para o contexto daquela 
escola (Escola Pará), via-me incentivada a buscar e a identificar que elementos de inclusividade estavam presentes naquela prática real reconhecida pela comunidade escolar como inclusiva.

É importante esclarecer que o termo inclusividade possui aqui uma aplicação semântica. A expressão práticas curriculares inclusivas apresenta a inclusão como qualidade da ação, portanto, adjetivo da prática, o que remeteria assim ao seu contrário (antônimo), que seria exclusão - lida como fracasso. Se inclusivas é adjetivo, e "o sufixo dade acrescido ao adjetivo, forma substantivo que expressa a ideia de estado, situação ou quantidade" (HERNADES, 2008), inclusividade, portanto, torna inclusivas um substantivo. Nesse sentido, a expressão práticas curriculares com elementos de inclusividade compreende a inclusão como coisa que se materializa em estado, situação ou quantidade. Portanto, a inclusão como substantivo (inclusividade) aplicado ao termo práticas curriculares sai de uma dimensão subjetiva (juízo de valor) para uma dimensão constitutiva, nesse sentido, revela materialidade. Materialidade essa que compõe a prática curricular daquela professora que teve sua prática reconhecida como inclusiva pela comunidade escolar da Escola Pará.

Conforme anunciei anteriormente, desloquei meu olhar da prática da professora para os argumentos da comunidade escolar da Escola Pará. A intenção era saber por que aquela prática com fortes marcas tradicionais e distantes do que orientam as discussões sobre inclusão era tida como inclusiva. Nesse sentido, foi necessário redefinir os sujeitos da pesquisa. Então, foram entrevistados: um coordenador pedagógico, uma coordenadora da Sala de Recursos Multifuncionais, uma professora da Sala de Recursos Multifuncionais e duas responsáveis por alunos com deficiência, além da própria professora indicada pela comunidade.

Com a categorização das entrevistas, pude identificar cinco elementos apontados pela comunidade que definiam a professora como tendo uma prática curricular inclusiva: a percepção do aluno em situação de deficiência como aluno; o aumento do tempo pessoal no tempo institucional/respeito do tempo institucional ao tempo pessoal; a frequência e a participação nos diversos espaços da escola; a apropriação de conhecimentos propriamente escolares; e o uso de artefatos escolares.

Contudo, observei que os aspectos apontados como inclusivos pela comunidade escolar da Escola Pará, aqui denominados elementos de inclusividade, não são característicos da escola inclusiva, mas característicos da escola, seja ela compreendida num feixe histórico longitudinal ou contíguo. Assim, tais argumentos me conduziram a pensar sobre a escola e a prática curricular desenvolvida na escola comum sem a preocupação de defini-las como inclusiva ou excludente. Foi a partir desses aspectos e da compreensão de que a prática é cultura objetivada (SACRISTAN, 1999), que a cultura escolar apareceu como uma categoria central para a análise dos elementos de inclusividade presentes na prática da Professora L.

Assim, se a partir da literatura da inclusão teria dificuldades em analisar tais elementos, inclusive porque a discussão sobre inclusão vem geralmente acompanhada da "elaboração" de uma pedagogia que pudesse garantir a sua efetividade, busquei a partir da cultura escolar compreender e analisar tais elementos.

Vale ressaltar que o que está subjacente à categoria cultura escolar não são os mecanismos de exclusão que foram se constituindo do decorrer da história da escola, mas a intenção de compreender a instituição escola como possuidora de uma cultura própria (FARIA FILHO, 2002).

Nesse sentido, cabe o cotejamento de duas perspectivas: a escola ideal - definida pela literatura e pela política da inclusão - e a escola real - cotidianamente vivida por sujeitos reais.

Contudo, a contradição escola ideal - escola real carrega uma intercessão. Retirados os mecanismos de exclusão-inclusão, sem reduzi-los a binarismos, excluir-incluir é referente a algo. A estar/não estar em algum lugar ou fazendo alguma coisa. É nessa linha de raciocínio que busco compreender os argumentos da comunidade escolar no reconhecimento da prática da professora $\mathrm{L}$ como inclusiva e, a partir daí, encontrar a síntese integrativa de uma perspectiva de escola e, mais especificamente, da prática que se propõe inclusiva. Síntese esta que pode ser encontrada pela compreensão da existência de uma cultura própria da escola, a cultura escolar, que ganha uma forma de representação no meio social e foi historicamente produzida. 
Existe uma frente de pesquisa que tem por objetivo discutir a cultura escolar a partir das práticas escolares, encontrando no cotidiano dessa instituição elementos de emergência da cultura. Para Gonçalves (2002, p. 1):

[...] será nesse cotidiano que as ações dos sujeitos serão inventadas ou reinventadas, gerando não somente as ações passivas de reprodução das imposições formais dos regulamentos e programas prescritos, mas sobretudo, desenvolvendo uma relação complexa de astúcias com tais imposições, com tramas de sociabilidades com seus pares e com outros sujeitos implicados nas relações mais extensas, seja no meio familiar, comunitário, ou outros. Relações de negociações, de conflitos, de burlas, de transgressões, de criação e de resistência.

Para Faria Filho et al (2004, p. 151):

[...] tais práticas produzidas pelos sujeitos no seu dia-a-dia escolar, também os produzem. Essas práticas têm sido concebidas [...] como maneiras de fazer peculiar dos sujeitos da escola e que ocorrem no interior do cotidiano escolar. Mas esse lugar ocupado por eles não tem sido entendido como um lugar próprio e, sim, como um lugar onde se desenvolvem táticas [...]

Táticas utilizadas como formas de luta contra a pressão das estratégias advindas das políticas, das instituições sociais. Certeau (2012, p. 93-95) assim diferencia estratégia de tática:

Estratégia é o cálculo (ou manipulação) das relações de força que se torna possível a partir do momento em que o sujeito de querer e poder (uma empresa, um exército, uma cidadã, uma instituição científica) pode ser isolado. A estratégia postula um lugar suscetível de ser circunscrito como algo próprio e a ser a base de onde se podem gerir as relações com uma exterioridade de alvos ou ameaças. [...]. Chamo tática a ação calculada que é determinada pela ausência de um próprio. [...]. A tática não tem por lugar senão o outro. E por isso deve jogar com o terreno que lhe é imposto tal como organiza a lei de uma força estranha. [...]. Em suma, a tática é a arte do fraco.

Essa leitura nos dá condições de pensar como as práticas curriculares desenvolvidas cotidianamente no interior da escola pelos diferentes sujeitos que as produzem experimentam e se constroem num processo de conflitos, tensões e negociações, levando à instabilidade das ações pedagógicas. Isso evidencia o não lugar dos sujeitos que produzem a cotidianidade da cultura escolar, mas também coloca e evidencia um lugar reclamado, existente, mas não reconhecido.

Tal perspectiva coloca-se como fundamental na compreensão dos elementos de inclusividade presentes numa prática curricular reconhecida como inclusiva por uma comunidade escolar, especialmente por esta articular a compreensão de que a instituição escolar possui uma cultura que Ihe é própria, aquela que tem sua marca e que a diferencia das culturas presentes em outras instituições sociais. O que por si demarca uma representação da sociedade sobre o que é e o que se faz (ou se deve fazer) na escola. Pois,

Concebido como produção histórica, o modelo escolar que se delineia se vai constituindo a partir de uma cultura que toma uma forma escolar própria, e que é caracterizada por dispositivos de normatização pedagógica e, sobretudo, de práticas dos agentes que se apropriam deles, por meio de estratégias e de táticas reveladoras de saberes socialmente acumulados que permitam que a identidade escolar seja construída e que os valores e comportamentos sejam difundidos. (GONÇALVES; FARIA FILHO, 2005, p. 50)

Vale dizer que compreender a cultura escolar como cultura própria da escola não a isola do contexto histórico, social, político e cultural que a cerca. Reconhece que ela não é apenas reprodutora de determinantes externos, mas também partícipe da produção desse contexto. Apropriando-me de Forquin (1993, p. 167), é possível afirmar que "a escola é também um mundo social, que tem suas características de vida próprias, seus ritmos e seus ritos, sua linguagem, seu imaginário, seus modos próprios de regulação e de transgressão, seu regime próprio de produção e de gestão de símbolos".

Num outro contexto e na busca de fazer uma análise da cultura escolar a partir das especificidades que a constituem uma cultura própria da escola, Faria Filho (2002) compreende a escola como um espaço/tempo de promoção de processos de escolarização. Para ele, a escolarização pode ser compreendida a partir de duas acepções: 
Num primeiro [sentido], escolarização pretende designar o estabelecimento de processos e políticas concernentes à "organização" de uma rede, ou redes, de instituições, mais ou menos formais, responsáveis pelo ensino elementar da leitura, da escrita, do cálculo e, o mais das vezes, da moral e da religião, seja pelo atendimento em níveis posteriores e mais aprofundados. $[\ldots]$.

Em outra acepção, estamos entendendo por escolarização o processo e a paulatina produção de referências sociais tendo a escola, ou a forma escolar de socialização e transmissão de conhecimentos, como eixo articulador de seus sentidos e significados. (FARIA FILHO, 2002, p. 16) (grifo meu)

Posteriormente, Faria Filho (2007) recupera os sentidos atribuídos à escolarização e acrescenta um terceiro: "aquele que nos remete mais diretamente ao ato ou efeito de tornar escolar, ou seja, o processo de submetimento de pessoas, conhecimentos, sensibilidades e valores aos imperativos escolares" (FARIA FILHO, 2007, p. 195).

Para o autor, "é justamente essa faceta da escolarização que, relacionada às outras duas, permite articular a escolarização com a configuração de um tipo específico de formação/organização cultural a que estamos denominando cultura escolar" (FARIA FILHO, 2007, p. 195)

Essa categoria, vinculada ao processo de escolarização, permite, segundo Faria Filho (2002, 2007) articular, descrever e analisar o complexo das relações que constituem os elementos-chaves da escola na composição do fenômeno educativo: os tempos, os espaços, os sujeitos, os conhecimentos e as práticas escolares.

Numa perspectiva das mezo abordagens, Faria Filho (2007, p. 195) define cultura escolar como:

A forma como em uma situação histórica concreta e particular são articuladas e representadas [sic], pelos sujeitos escolares, as dimensões espaço-temporais do fenômeno educativo escolar, as sensibilidades e os valores a serem transmitidos e a materialidade e os métodos escolares. (grifo meu)

É exatamente essa articulação e representação em uma situação histórica que me coloca diante da necessidade de analisar o que define uma prática inclusiva para uma determinada comunidade escolar e por que essa prática parece tão distante daquilo que é definido pelas políticas e pela literatura especializada sobre a inclusão da pessoa em situação de deficiência na classe comum das escolas regulares.

Nesse sentido, e compreendo que há um distanciamento entre a percepção da comunidade escolar e a lente da literatura especializada sobre a prática objeto de investigação desta pesquisa, busquei encontrar as inflexões/deslocamentos da prática da professora L por meio da cultura escolar, não no sentido de redimir ou ovacionar o trabalho desenvolvido pela professora, mas na intenção de analisar por que a produção dessa forma de "fazer inclusão" é reconhecida como efetivamente inclusiva, mesmo que distante daquilo que se espera como prática inclusiva.

As cinco categorias emergentes das falas dos sujeitos (citadas anteriormente) estão diretamente vinculadas à dimensão complementar do qual Gonçalves (2002) chama atenção nos estudos sobre cultura escolar: a ação cotidiana. De forma mais precisa, as categorias apresentadas são consoantes aos elementos-chaves do fenômeno educativo que irão configurar a cultura escolar, definidos por Faria Filho (2002): o tempo, o espaço, os sujeitos, os conhecimentos, as práticas.

Assim, no contexto da educação inclusiva, especialmente do reconhecimento de uma prática curricular, com fortes marcas características de uma educação tradicional, como inclusiva, tentei buscar compreender que, a despeito dos mecanismos de exclusão e dentro dos seus limites, o complexo fenômeno educativo constituidor da cultura escolar - sujeitos, tempo, espaço, conhecimento e práticas - conforma, de maneira articulada, uma representação compartilhada socialmente sobre o que é e o que se faz na escola.

Diante dos dados obtidos no lócus de pesquisa, percebi que o que está subjacente a esse reconhecimento é a possibilidade que a prática dessa professora traz de construir mecanismos de aproximação - inclusão - desses sujeitos a esses elementos que constituem o imaginário de "estar" na escola e "estudando", mesmo valendo-se de estratégias pouco inovadoras. 
Ora, nesse cenário a participação garante ao aluno em situação de deficiência estar na escola e usufruir dos seus diferentes tempos e espaços. A apropriação está na possibilidade desse aluno aprender e se desenvolver por meio de diferentes experiências/vivências que são "típicas" da instituição escolar. A produção evidencia que os alunos em situação de deficiência têm colaborado/ estimulado a construção de uma "nova" cultura escolar.

Nesse sentido, se eu retomar o foco para a prática curricular da professora $L$ me valendo dos argumentos que a comunidade escolar da Escola Pará me apresentou, os elementos de inclusividade, dos fundamentos da literatura da inclusão e da categoria cultura escolar, é possível afirmar que a prática curricular inclusiva se produz na intercessão entre os elementos do passado - a representação compartilhada socialmente sobre a escola e a cultura própria que esta produziu -, com os elementos do futuro - os avanços que se espera que a escola conquiste -, e os elementos do presente - as possibilidades reais entre o passado e o futuro vividas no cotidiano por sujeitos reais.

\section{CONSIDERAÇÕES FINAIS}

Compreender a inclusão numa interface com a cultura escolar coloca uma lente naquilo que algumas vezes é negligenciado: o fazer cotidiano na escola e seus impactos nas expectativas daqueles que recebem a educação promovida por essa instituição.

O modelo de escola inclusiva e de prática curricular inclusiva, de acordo com os documentos do Ministério da Educação, deve romper com a cultura escolar. Por outro lado, para a comunidade escolar da Escola Pará, a escola inclusiva se desenvolveria na continuidade da cultura escolar instituída, ou seja, aquilo que é própria da escola sendo também disponível aos alunos em situação de deficiência.

Ao que parece existe um distanciamento muito forte entre essas duas perspectivas, o que poderia ser minimizado pela síntese entre a perspectiva prescrita da cultura escolar (estratégias) e a cultura escolar instituída e materializada nas práticas curriculares dos professores (táticas).

Diante dessas afirmativas é possível fazer a seguinte inferência: enquanto a política curricular inclusiva for compreendida como um modelo a ser seguido e encapsulado na escola, esta e suas práticas continuarão sendo definidas como fracassadas.

Contudo, se tomarmos por base a articulação entre os dados de campo oriundos desta pesquisa e os anseios das políticas curriculares inclusivas e dos especialistas dessa área, a prática curricular inclusiva não deveria se configurar no rompimento da cultura escolar existente, mas a ressignificação da mesma, (re)inventada na síntese dos deslocamentos dos eixos articuladores da cultura escolar, sem, no entanto, descaracterizar o seu próprio, promovendo, assim, a tão difícil síntese reproduçãoinovação das práticas educativas.

\section{REFERÊNCIAS}

AGÊNCIA EUROPEIA PARA O DESENVOLVIMENTO DA EDUCAÇÃO ESPECIAL. Educação Inclusiva e Práticas de Sala de Aula nos $2^{\circ}$ e $3^{\circ}$ Ciclos do Ensino Básico. Relatório Síntese, 2005. Disponível em: www.european-agency.org. Acesso em: ago.2012.

CASTRO, Adriano Monteiro. A prática pedagógica do professor de ciências e a inclusão do aluno com deficiência visual na escola pública. São Paulo: USP, 2002. (Dissertação de Mestrado)

CERTEAU, Michel de. A invenção do cotidiano: 1 artes de fazer. 18. ed. Trad. Ephraim Ferreira Alves. Petrópolis, RJ: Vozes, 2012.

FARIA FILHO, Luciano et al. A cultura escolar como categoria de análise e como campo de investigação na história da educação brasileira. In: Educação e Pesquisa. São Paulo, v.30, n.1, p. 139-159, jan./ abr.2004.

FARIA FILHO, Luciano M.; GONÇALVES. História das culturas e das práticas escolares: perspectivas e desafios teórico-metodológicos. In: SOUSA, Rosa F. de; VALDEMARIN, Vera T. (Orgs.). A cultura escolar em debate: questões conceituais, metodológicas e desafios para a pesquisa. Campinas, SP: Autores Associados, 2005, p. 31-58. 
FARIA FILHO, Luciano M. Escolarização, culturas e práticas escolares no Brasil: elementos teóricometodológicos de um programa de pesquisa. In: LOPES, Alice C.; MACEDO, Elizabeth (Orgs.). Disciplinas e integração curricular: história e políticas. Rio de Janeiro: DP\&A, 2002, p. 13-36.

Escolarização e cultura escolar no Brasil: reflexões em torno de alguns pressupostos e desafios. In: BENCOSTTA, Marcus L. (Org.). Culturas escolares, saberes e práticas educativas: itinerários históricos. São Paulo: Cortez, 2007, p. 103-211.

FORQUIN, Jean-Claude. Escola e Cultura: as bases sociais e epistemológicas do conhecimento escolar. Trad. Guacira Lopes Louro. Porto Alegre: Artes Médicas, 1993.

GONÇALVES, Irlen A. A produção da cultura escola em Minas Gerais. Práticas de professoras e alunos da escola primária. II Congresso da SBHE. 2002. Disponível em: www.sbhe.org.br/novo/congressos/ cbhe2/pdfs/Tema3/0323.pdf. Acesso em: out./2012.

HERNANDES, Paulo. Emprego do sufixo "-dade". 2008. Disponível em: http://www.paulohernandes. pro.br/. Acesso em: jun/2012

MIOTTO, Ana Cristina Felipe. A prática curricular e suas implicações no trabalho com os educandos com deficiência visual: avanços e impasses na inclusão. Minas Gerais: PUC, 2010. (Dissertação de mestrado)

PLETSCH, Márcia Denise. Repensando a inclusão escolar de pessoas com deficiência mental: diretrizes políticas, currículo e práticas pedagógicas. Rio de Janeiro: UERJ, 2009. (Tese)

SACRISTÁN, J. Gimeno. Poderes instáveis em educação. Porto Alegre: ARTMED, 1999.

VICENT, Guy; LAHIRE, Bernard; THIN, Daniel. Sobre a história e a teoria da forma escolar. In: Educação em Revista [on-line]. 2001, n.33, pp. 07-47. Disponível em: http://educa.fcc.org.br/pdf/edur/n33/ n33a02.pdfi. Acesso em: ago./2012.

ZABALA, Antoni. A prática educativa: como ensinar. Trad. Ernani F. da F. Rosa. Porto Alegre: ArtMed, 1998.

Artigo recebido em 31/10/2013

Aprovado em 21/11/2013 